This item was submitted to Loughborough's Research Repository by the author.

Items in Figshare are protected by copyright, with all rights reserved, unless otherwise indicated.

\title{
Evolving hierarchical gene regulatory networks for morphogenetic pattern formation of swarm robots
}

PLEASE CITE THE PUBLISHED VERSION

http://dx.doi.org/10.1109/CEC.2014.6900365

PUBLISHER

(C) IEEE

VERSION

AM (Accepted Manuscript)

LICENCE

CC BY-NC-ND 4.0

\section{REPOSITORY RECORD}

Oh, Hyondong, and Yaochu Jin. 2015. "Evolving Hierarchical Gene Regulatory Networks for Morphogenetic Pattern Formation of Swarm Robots". figshare. https://hdl.handle.net/2134/17857. 


\title{
Evolving Hierarchical Gene Regulatory Networks for Morphogenetic Pattern Formation of Swarm Robots
}

\author{
Hyondong Oh and Yaochu Jin
}

\begin{abstract}
Morphogenesis, the biological developmental process of multicellular organisms, is a robust self-organising mechanism for pattern formation governed by gene regulatory networks (GRNs). Recent findings suggest that GRNs often show the use of frequently recurring patterns termed network motifs. Inspired by these biological studies, this paper proposes a morphogenetic approach to pattern formation for swarm robots to entrap targets based on an evolving hierarchical gene regulatory network (EH-GRN). The proposed EH-GRN consists of two layers: the upper layer is for adaptive pattern generation where the GRN model is evolved by basic network motifs, and the lower layer is responsible for driving robots to the target pattern generated by the upper layer. Obstacle information is introduced as one of environmental inputs along with that of targets in order to generate patterns adaptive to unknown environmental changes. Besides, splitting or merging of multiple patterns resulting from target movement is addressed by the inherent feature of the upper layer and the $k$-means clustering algorithm. Numerical simulations have been performed for scenarios containing static/moving targets and obstacles to validate the effectiveness and benefit of the proposed approach for complex shape generation in dynamic environments.
\end{abstract}

\section{INTRODUCTION}

Developing self-organising multi-robot systems has been actively researched in recent decades due to its attractive properties such as robustness to failures and damages of the part of systems, adaptability to unknown environments and cost efficiency. Considering large-scale but relatively simple swarm robots in a real world, many constraints such as limited computation, communication and sensing capabilities make the development of autonomous swarming system challenging. Specific applications for multi-robot systems under consideration include, but are not limited to, search and rescue [1], [2], collective transportation and construction [3], [4], deployment of sensor networks [5] and formation flying of micro-UAVs [6] and small satellites [7].

In particular, considerable attention has been paid to multirobot pattern formation as a basic functionality for achieving above missions, which can be largely divided into four categories: 1) behaviour-based control in which a desired set of behaviours with relative importance is implemented onto individual robots [8], [9]; 2) leader-follower structures where the leaders are identified and the followers are supposed to follow the leaders with rigid or relaxed formation constraints [10], [11]; 3) potential field in which the robot moves along the gradient of a potential field, which is a sum of attractive

Hyondong Oh and Yaochu Jin are with the Department of Computing, University of Surrey, Guildford, Surrey, GU2 7XH UK (email: \{h.oh,yaochu.jin\}@surrey.ac.uk).

This work was funded by the European Commission 7th Framework Program, Project No. 601062, SWARM-ORGAN. and repulsive forces [12], [13]; and 4) biologically-inspired approaches where robots are controlled by a model inspired from a biological system, such as a pheromone/hormone model [14], [15] or a gene regulatory network (GRN) model [16], [17].

A significant limitation of most existing pattern formation algorithms for multi-robot systems is that the target shape needs to be predefined and forms only a small number of simple geometric shapes, which may become inadequate for dealing with unknown complex environments. To alleviate this problem, Beta and Kumar [18] proposed a formation control method for a large number of robots based on an abstraction of the team to a small dimensional manifold independent of the number of robots. In this work, although target shapes can be adaptable to environmental changes by changing the shape size (as demonstrated with a narrow tunnel passing scenario), only two simple shapes are considered and the final formation results in the loose distribution of robots inside the shape. Cheah et al. have introduced a region-based shape control for robot swarms based on potential field, where each robot in the group stays within a moving region while maintaining a minimum distance from each other [19], [20]. They considered various shapes as combination of inequality functions with convergence analysis. However, the complexity of the target shapes is still limited, and the target shape must be predefined. On the other hand, Swarm Chemistry, a computational model of particle swarms following certain kinetic rules, is introduced in [21] for designing various complex patterns. In this framework, however, average velocity of nearby particles is required, which is difficult to obtain in practice, and arbitrary patterns arise as emergent phenomenon from interactions between swarm particles rather than being able to generate task-driven or adaptable complex shapes.

Biologically-inspired approaches provide us with more promising and flexible ways to generate an adaptive and robust pattern to cope with unknown environmental changes and disturbances [22]-[24]. To this end, a hierarchical GRN (H-GRN) concept is introduced for target entrapping [25] based on the fact that hierarchy in GRNs plays a central role in the evolution of developmental GRNs, resulting in better adaptability and evolvability [26]. In this work, the first layer is responsible for adaptive pattern generation, while the second is a control mechanism that drives the robots on to the generated pattern. The generated pattern is shown to be adaptable to dynamic targets and robust to robot failures. However, in order to avoid incoming obstacles, the algorithm relies only on a repulsive avoidance 
scheme using a diffusion term of robots and obstacles in a GRN model. This leads to the situation while some of robots remain at their corresponding positions to entrap the targets, robots closer to obstacles move away from their desired entrapping positions to avoid obstacles. In other words, although the H-GRN algorithm generates adaptive patterns according to the position of targets, this pattern is not adaptable to obstacles or any other environmental inputs as it uses a fixed GRN structure for pattern generation. To address unknown environmental inputs more generically, a network motifs based GRN (NM-GRN) model is proposed, which evolves some predefined network motifs-frequently recurring network patterns-as basic building blocks [27]. The NM-GRN is used to automatically generate different yet suitable shapes for robots to traverse an unknown dynamic environment.

Inspired by the benefit of these biological approaches, this paper proposes a morphogenetic approach to target entrapping based on an evolving hierarchical gene regulatory network (EH-GRN). The EH-GRN consists of two layers: the upper layer is for adaptive pattern generation where the GRN model is evolved by basic network motifs with genes and environmental inputs, and the lower GRN layer is for driving robots to the target pattern generated by the upper layer. This GRN framework enables us to generate patterns adaptive and robust to environmental changes. Particularly, by introducing obstacles as one of environmental inputs along with targets, we address the weakness of pattern from the previous H-GRN [25] not being adaptable to obstacles in that a target entrapping pattern itself changes as an obstacle approaches. This allows all the robots in the swarm to continue both of their missions: entrapping targets and avoiding obstacles simultaneously. In addition, the proposed evolving GRN framework improves the flexibility of the pattern generation to be applied for various tasks as it has an open structure of gene regulations in a GRN model to some extent. Separability aspect (splitting or merging) of multiple patterns resulting from target movement is addressed by the inherent feature of the upper layer and the $k$-means clustering algorithm [28].

The rest of this paper is structured as follows. Section II presents a problem statement and biological background for morphogenetic swarm robotics and basic network motifs. Section III introduces the EH-GRN model with an evolving strategy, consisting of an upper layer for pattern generation and a lower layer for pattern formation control, where the dynamics of the two GRNs are different. Section IV presents numerical simulation results from scenarios containing multiple stationary and moving targets and an obstacle. Conclusions and future work are given in Section V.

\section{Problem Statement AND BACKGRound}

\section{A. Problem Statement}

This paper considers the problem of entrapping dynamic targets while avoiding obstacles using a two-layer hierarchical gene regulatory network with a swarm of robots.
Based on the location of targets and obstacles, an adaptive pattern is generated by the upper layer, and the robots are deployed into the generated pattern by the lower layer GRN dynamics. Similar to [25], we distinguish between organising robots that can detect at least one target and are responsible for the pattern generation, and non-organising robots that have not yet detected any target. The non-organising robots follow their neighbouring organising robots while obtaining information of a target until they detect it themselves. Several assumptions are made in this study: i) the robots can localise themselves in a local coordinate system with their onboard sensors and local communication among the robots; ii) all targets in the region of interest can be detected by at least one robot, and coverage or search aspect is not considered in this study; iii) the movement of robots is much faster than the targets and the obstacles, iv) all robots have a limited sensing and communication range, and $\mathrm{v}$ ) communication bandwidth is wide enough to transmit required data between robots.

\section{B. Morphogenetic Swarm Robotics}

The past decade has witnessed rapid technical and theoretical advances in evolutionary developmental biology and systems biology in understanding molecular and cellular mechanisms that control the biological morphogenesis [17]. In particular, morphogenetic swarm robotic systems deal with the self-organisation of swarm (a large number of homogeneous and simple) robots using genetic and cellular mechanisms underlying the biological early morphogenesis [14], [16], [29]. Here, biological morphogenesis is the biological process in which cells divide, grow, and differentiate, and finally resulting in the mature morphology of a biological organism. Morphogenesis is under the governance of developmental gene regulatory networks (GRNs) and the influence of the environment where GRNs are models of genes and the interaction of gene products that describe the gene expression dynamics [30]. The environment includes concentration of gradients of substances known as morphogens which are responsible for cell specialisation and migration. Morphogen gradients can be present in the environment of the fertilised cell or generated by a few cells known as organisers [31].

In this study, we seek to develop a morphogenetic swarm robotic system exploiting the concept of a morphogen gradient and GRNs. The basic idea in applying genetic and cellular mechanisms in biological morphogenesis to control of swarm robots is to establish a metaphor between a cell and a robot. In this metaphor, each cell can be seen as a single robot where protein concentrations in the cell correspond to the internal states and the location of the robot. Each protein can provide the following three functions: i) auto-regulation (regulate the expression of the gene that produces the protein, thus adjusting the robot's behaviour); ii) interaction with a certain morphogen gradient in the environment (in relation to the target entrapping shape in this paper); iii) diffusion into environment to avoid collisions among the robots. 


\section{Basic Network Motifs}

Recent biological research findings suggest that network motifs, patterns of inter-connections, occurs in real complex networks at numbers significantly higher than those in randomised networks [32]. This frequently recurring network motifs can also be found in GRNs of a multi-cellular organism [33]. Inspired by these findings, this study utilises five basic regulations: positive, negative, AND, OR, and XOR regulations as the basic network motifs to construct GRN networks.

Firstly, a positive (or negative) regulation is defined as gene $X$ activates (or inhibits) or pose a positive (or negative) feedback to gene $Y$. The mathematical description of the positive regulation from $X$ to $Y$ can be represented as:

$$
\begin{aligned}
\frac{d y}{d t} & =-y+\operatorname{sig}(x, \theta) \\
\operatorname{sig}(x, \theta) & =\frac{1}{1+e^{-k(x-\theta)}}
\end{aligned}
$$

where $x$ and $y$ represent the expression level of gene $X$ and $Y$, respectively, and $\theta$ is a regulatory parameter for the gene expression. Similarly, the negative regulation is represented as:

$$
\frac{d y}{d t}=-y+(1-\operatorname{sig}(x, \theta))
$$

In the AND-regulation, gene $Y$ expresses only if both gene $X_{1}$ and $X_{2}$ express, as defined by:

$$
\frac{d y}{d t}=-y+\operatorname{sig}\left(g_{1} \cdot g_{2}, \theta\right)
$$

where $g_{1}$ and $g_{2}$ are the expression levels of gene $X_{1}$ and $X_{2}$, respectively, and the expression level of each gene is governed by a positive or negative regulation with a regulatory parameter $\theta_{1}$ or $\theta_{2}$. In the OR-regulation, gene $Y$ expresses if either gene $X_{1}$ or $X_{2}$ expresses, as defined by:

$$
\frac{d y}{d t}=-y+\operatorname{sig}\left(g_{1}+g_{2}, \theta\right)
$$

Lastly, in the XOR-regulation, gene $Y$ expresses only if gene $X_{1}$ and $X_{2}$ have disparate expression levels, as defined by:

$$
\frac{d y}{d t}=-y+\operatorname{sig}\left(g_{1} \cdot\left(1-g_{2}\right), \theta\right)+\operatorname{sig}\left(\left(1-g_{1}\right) \cdot g_{2}, \theta\right)
$$

Thus, if gene $X_{1}$ expresses and gene $X_{2}$ does not express (or the other way round), gene $Y$ expresses. These regulations will be the basic building blocks of our GRN structure, and a regulatory parameter $\theta$ will be optimised by an evolutionary algorithm.

\section{Evolving H-GRN Using Network Motifs}

General structure of the proposed EH-GRN using network motifs to be evolved is illustrated in Fig. 1. Firstly, the upper layer of the EH-GRN is for adaptive pattern generation where the GRN model is evolved by the combination of basic network motifs with genes and environmental inputs. In Fig. 1, $p_{1}$ and $p_{2}$ represent the protein concentrations produced by the environmental inputs (i.e. target and obstacle if any) and diffused spatially. These will serve as the inputs to the upper

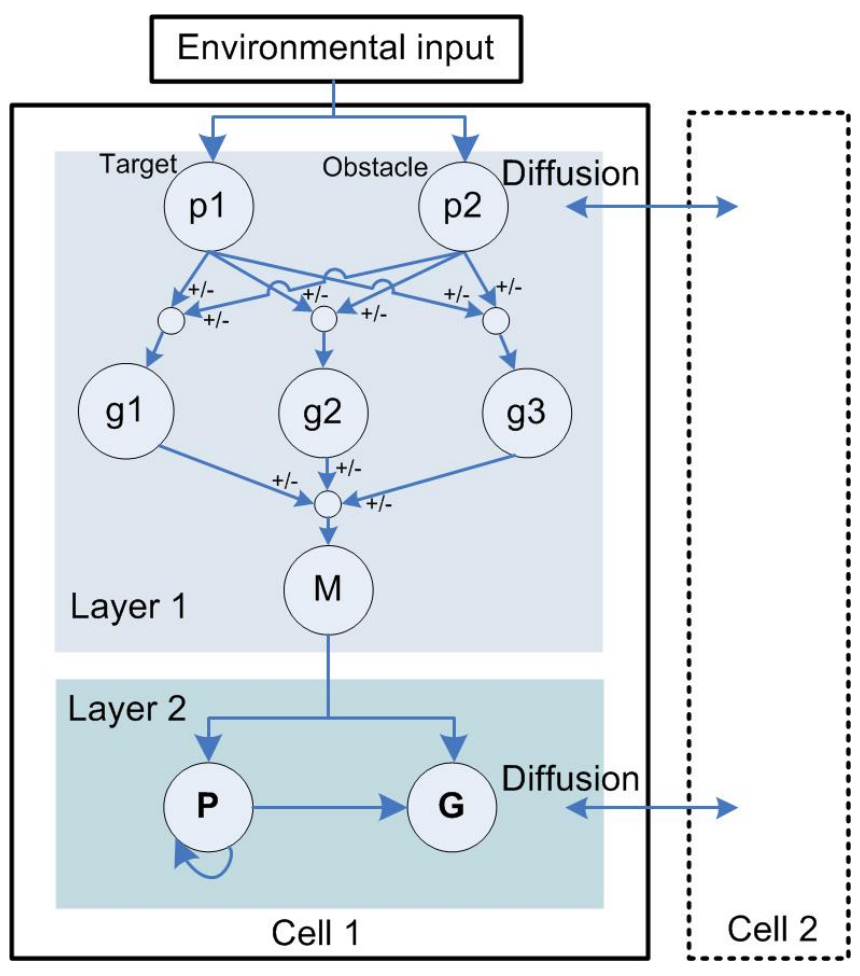

Fig. 1. Illustration of an evolving two-layer H-GRN structure for target entrapping.

GRN layer and activate gene $g_{1}, g_{2}$ and $g_{3}$. In particular, the concentration of $M$ takes the role of morphogen to form the desired shapes around targets which will be transmitted to the lower layer. Note that the dynamics of the GRN in the upper layer is activated only in the organising robots that are able to detect targets, while the non-organising robots simply follow the movement of neighbouring organising robots. By introducing obstacles as one of environmental inputs along with targets, a target entrapping pattern itself changes as an obstacle approaches for all the robots to continue both of their missions: entrapping and avoidance at the same time. As a preliminary study, this work only evolves the upper layer with the fixed number of genes. However, the entire structure including the lower layer and varying number of genes can be used with the same methodology exploiting basic network motifs introduced in this paper.

Once the target pattern is generated by the upper layer, it will function as the input of the lower layer to trigger its dynamics. The lower layer is responsible for pattern formation control guiding all robots on to the generated pattern and maintaining a minimum distance between the robots at all times. These functions are realised by another GRN dynamics where proteins $\mathbf{G}$ and $\mathbf{P}$ are used to represent the current position and internal state of the robot. With the diffusion of $p_{1}, p_{2}$ and $\mathbf{G}$, the robots can share the information on the target and the obstacle and maintain the desired minimum distance amongst robots in order to avoid robot to robot collisions. 


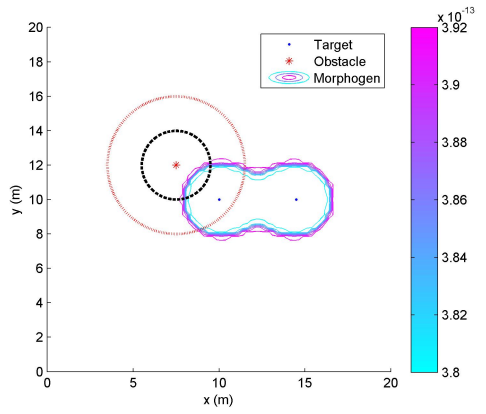

(a) Morphogen gradient sapce

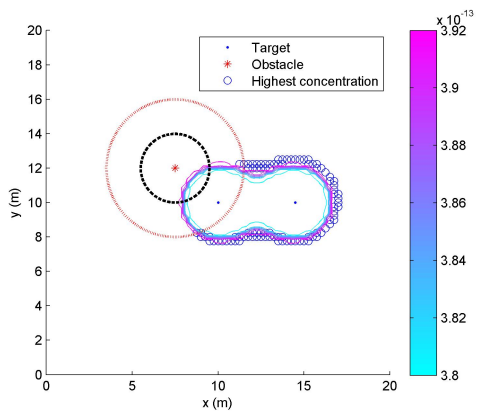

(b) Highest concentration

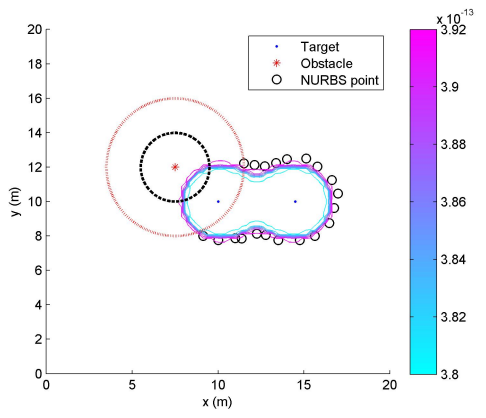

(c) NURBS points

Fig. 2. NURBS representation of a target entrapping pattern.

\section{A. Upper Layer of the EH-GRN}

The generation process of an adaptive target entrapping pattern consists of two steps: i) forming a morphogen gradient space according to the evolving GRN model and extracting candidate points whose gradient value is higher than a threshold and ii) representing candidate points with a Non Uniform Rational B-Splines (NURBS) [34] to generate target pattern for robots to follow, as illustrated in Fig. 2.

A morphogen gradient space is first generated by the regulation parameters and environment inputs using the upper layer as shown in Fig. 2(a), and the points whose gradient value is higher than a certain percentage of the maximum value are selected as candidate points in Fig. 2(b). Requirement for the desired morphogen gradient space for target entrapping is to have as many candidate points as possible i) satisfying not too close $\left(d_{\min }\right)$ to or far away $\left(d_{\max }\right)$ from targets and ii) maintain a minimum distance $\left(d_{\text {min }}^{\text {obs }}\right)$ to the obstacle. The fitness function for the evolutionary algorithm can then be set up as:

$$
\begin{aligned}
f= & \sum_{i=1}^{N_{p}} \sum_{j=1}^{N_{t}} \frac{\operatorname{sig}\left(d_{p t}^{i j}, d_{\max }, k_{1}\right)+\operatorname{sig}\left(d_{\min }, d_{p t}^{i j}, k_{2}\right)-A_{i j}}{N_{p} N_{t}} \\
& +\sum_{i=1}^{N_{p}} \sum_{k=1}^{N_{o}} \frac{\operatorname{sig}\left(d_{\min }^{\text {obs }}, d_{p o}^{i k}, k_{3}\right)}{N_{p} N_{o}}
\end{aligned}
$$

where

$$
A_{i j}= \begin{cases}1, & \text { if } d_{\min } \leq d_{p t}^{i j} \leq d_{\max } \\ 0, & \text { otherwise }\end{cases}
$$

and $N_{p}, N_{t}$ and $N_{o}$ represent the number of candidate points, targets and obstacles, respectively. $d_{p t}^{i j}$ denotes a distance between $i$ th candidate point and $j$ th target, $d_{p o}^{i k}$ denotes a distance between $i$ th candidate point and $k$ th obstacle.

For the upper layer, there are 26 parameters to be optimised: $\theta_{i}(i=1, \cdots, 13)$ for a regulatory parameter between environmental inputs $p_{i}(i=1,2)$ and genes $g_{i}(i=1,2,3)$ and genes and morphogen gradient $M$ as introduced in Section II.C and shown in Fig. $1 ; t_{i}(i=1, \cdots, 9)$ for a positive or negative regulation; and $c_{i}(i=1, \cdots, 4)$ for AND or OR regulation. XOR regulation is not used in this work for the simplicity. $t_{i}$ can be 0 (positive regulation) or 1 (negative regulation), and also $c_{i}$ can be 0 (AND regulation) and 1 (OR regulation). For instance, if $t_{1}=1, t_{2}=0$, and $c_{1}=0$, then the partial dynamics between target $p_{1}$, obstacle $\left(p_{2}\right)$ and gene $1\left(g_{1}\right)$ can be described as:

$$
\begin{aligned}
\frac{d g_{11}}{d t} & =-g_{11}+\operatorname{sig}\left(p_{1}, \theta_{1}\right) \\
\frac{d g_{12}}{d t} & =-g_{12}+\left(1-\operatorname{sig}\left(p_{2}, \theta_{2}\right)\right) \\
\frac{d g_{1}}{d t} & =-g_{1}+\operatorname{sig}\left(g_{11} \cdot g_{12}, \theta_{7}\right)
\end{aligned}
$$

where $g_{11}$ and $g_{12}$ are internal genes between environmental inputs and $g_{1}$. Entire upper layer dynamics of the EH-GRN can be constructed with a similar manner using defined 26 parameters.

The covariance matrix adaptation evolution strategy (CMA-ES) [35] is used to optimise the regulatory parameters. Since CMA-ES performs optimisation in a continuous domain, discrete parameters such as $t$ (positive/negative regulation) and $c$ (AND/OR logic) need to be transformed into continuous values. In this work, if the parameter is less than 0.5 , it is considered as 0 , and otherwise 1 . The population size is set to 200 with 100 for parent and 200 for offspring, and the CMA-ES was run for 50 generations. Although the population size can also be determined based on the search dimension in the general CMA-ES algorithm, in this simulation, as there may exist many local optima due to the unconventional fitness formulation (such as extracting candidate points), a relatively big size is used. $\theta_{7}, \theta_{8}, \theta_{9}$ and $\theta_{13}$ are assigned to be real numbers from 0 to 2 and the rest of parameters to be real numbers from 0 to 1 . The resulting evolved structure of the upper layer is shown in Fig. 3 with the following parameter values: $\theta_{1}=0.0891, \theta_{2}=0.2764$, $\theta_{3}=0.0783, \theta_{4}=0.8363, \theta_{5}=0, \theta_{6}=0, \theta_{7}=1.1956$, $\theta_{8}=0.2957, \theta_{9}=0.8352, \theta_{10}=0.4230, \theta_{11}=0.3256$, $\theta_{12}=0.3226$, and $\theta_{13}=1.4299$.

In Fig. 3, the morphogen $M$ that defines a gradient space for the target pattern is regulated by: i) $g_{1}$ activated by both $p_{1}$ and $p_{2}$, which finally inhibits $M$, ii) $g_{2}$ inhibited by $p_{1}$ and $p_{2}$, which activates $M$, and iii) $g_{3}$ activated by $p_{1}$ and inhibited by $p_{2}$. Thus, gene $g_{1}$ can be considered making the target pattern being not too close to both targets 


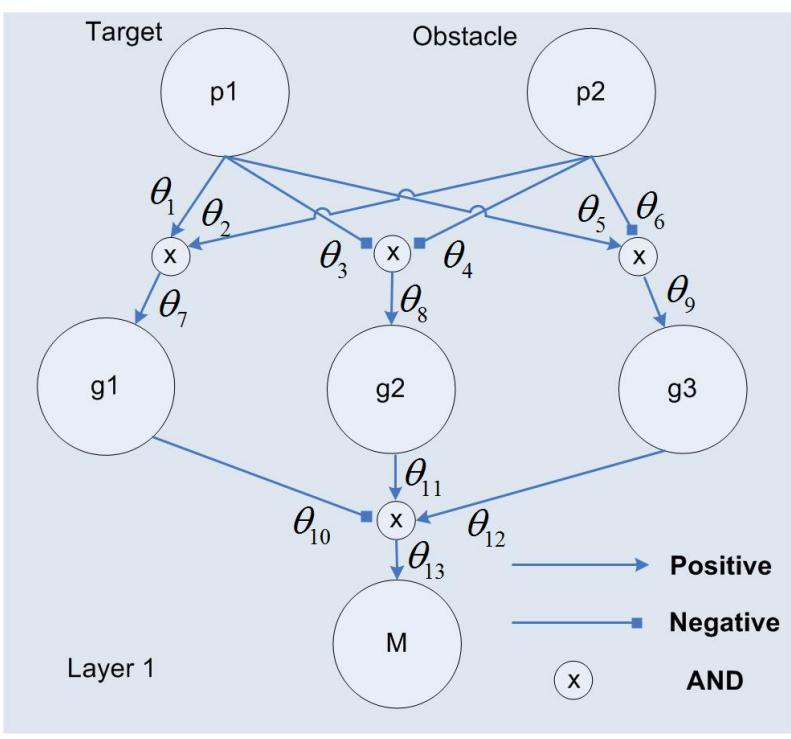

Fig. 3. Illustration of an evolved two-layer H-GRN structure for target entrapping.

and obstacles and $g_{2}$ is for target pattern not far way from both environmental inputs. The combinatory regulation of gene $g_{1}$ with a regulatory parameter $\theta_{10}=0.4230$ and $g_{2}$ with $\theta_{11}=0.3256$ to $M$ makes the target pattern a band of circle as shown in Fig. 2(b). Lastly, gene $g_{3}$ works as activating a morphogen gradient $(M)$ value when there is target expression and inhibiting it when there is obstacle expression, adding one more design dimension to the target shape.

Note that, as the targets move away from or get closer to each other, the number of entrapping patterns can be varied. Thus, this pattern separability aspect is addressed using $k$-means clustering algorithm to group candidate points based on attributes into pre-defined $k$ number of groups [28]. The grouping is done by minimising the sum of squares of distances $J$ between points and the corresponding cluster centroid. As there is no initial knowledge on the number of patterns, the number of cluster $k$ starts from one and increases by one while comparing the cost $J$ in order to find the best $k$.

The extracted candidate points whose gradient value is higher than the certain percentages of the maximum value from a morphogen gradient space $M$ are then used to construct a target entrapping pattern using Non Uniform Rational B-Splines (NURBS) [34] to be used by the EHGRN lower layer. The NURBS is a mathematical model commonly used in computer graphics and structural design for generating and representing curves and surfaces. NURBS is considered in the study since it can offer two unique features for pattern generation. First, it provides a common mathematical form for both analytical and free-form shapes. Second, it is a parameterised representation that is independent of an absolute coordinate system; once the parameter in the NURBS curve is fixed, a corresponding point on the NURBS curve can be determined without a global coordinate system. A NURBS curve can be defined as a combination of a set of piecewise rational basis functions with $n+1$ control points $\mathbf{p}_{i}$ and the associated weights $w_{i}$ as [34]:

$$
\mathbf{C}(u)=\frac{\sum_{i=1}^{n} \mathbf{p}_{i} w_{i} B_{i, k}(u)}{\sum_{j=1}^{n} w_{j} B_{i, k}(u)},
$$

where $n$ is the number of control points, $u$ is a parametric variable, and $B_{i, k}(u)$ are $\mathrm{B}$-spline basis functions where $i$ corresponds to the $i$ th control point and $k$ to the degree of the basis function. More details on the NURBS can be found in [34]. The generated NURBS is segmented to provide desired points for the robots to follow as shown in Fig. 2(c); this process will be explained in the next section.

\section{B. Lower Layer of the EH-GRN}

The lower layer of the EH-GRN is for guiding and controlling robots to the corresponding patterns generated by the upper layer. The GRN in the lower layer adopts a feed-forward loop which is the most commonly occurring GRN network motifs as found in [32]. As this network motif is shown to be efficient enough for robot guidance in [25], [36], [37], we use the same GRN dynamics as in our previous work, which was a modified version of a GRN model used in [38]:

$$
\begin{aligned}
\frac{d \mathbf{G}_{i}}{d t} & =-a \mathbf{z}_{i}+m \mathbf{P}_{i} \\
\frac{d \mathbf{P}_{i}}{d t} & =-c \mathbf{P}_{i}+r f\left(\mathbf{z}_{i}\right)+b \mathbf{D}_{i}
\end{aligned}
$$

where $i \in\left\{1, \cdots, N_{o}\right\}$ is the index of an organising robot, and protein types $\mathbf{G}_{i}$ and $\mathbf{P}_{i}$ correspond to a 2-D position and internal state vector of robot $i$, respectively. $a, m, c, r$ and $b$ are constants to be optimised depending on the objectives of the task. $\mathbf{D}_{i}$ represents the concentration of protein $\mathbf{G}$ diffused out of the cell, indicating the density of robots and obstacles in the neighbourhood:

$$
\mathbf{D}_{i}=\sum_{j=1}^{n_{i}} \mathbf{D}_{i}^{j},
$$

where $n_{i}$ denotes the number of robots in the neighbourhood of robot $i$, and $\mathbf{D}_{i}^{j}$ represents the diffused protein concentration vector from robot $j$ :

$$
\mathbf{D}_{i}^{j}=\frac{\mathbf{G}_{i}-\mathbf{G}_{j}}{\left\|\mathbf{G}_{i}-\mathbf{G}_{j}\right\|} .
$$

The diffusion process is activated only when the distance to the neighbour is less than a threshold $r_{n}$. This distance varies according to the target shape and the number of robots in the environment. In order to embed the 2-D target shape into the regulatory dynamics, $f\left(\mathbf{z}_{i}\right)$ is defined as the following sigmoid function:

$$
f\left(\mathbf{z}_{i}\right)=\frac{1-e^{\alpha \mathbf{z}_{i}}}{1+e^{\alpha \mathbf{z}_{i}}},
$$

where $\mathbf{z}_{i}$ represents the maternal morphogen gradient at the robot's current position, and $\alpha>0$ determines the slope of the sigmoid function. This $\mathbf{z}_{i}$ regulates the concentration of 
both proteins $\mathbf{G}$ and $\mathbf{P}$ in the GRN dynamics as in Eq. (14) so that robots could form a desired shape, defined as:

$$
\mathbf{z}_{i}=\mathbf{G}_{i}-\mathbf{C}\left(u_{i}\right)
$$

where $h_{i}$ is the desired target shape on which the robots need to be deployed defined by the upper layer, and $u_{i}$ should satisfy the following condition:

$$
u_{i}=\arg \min _{0 \leq u \leq 1}\left\|\mathbf{G}_{i}-\mathbf{C}\left(u_{i}\right)\right\|
$$

Since it is difficult to have an analytic function to represent of the NURBS $\mathbf{C}\left(u_{i}\right), u$ is segmented in the range of $[0,1]$ by the number of robots in the environment as $u_{d}=\{0,1 /(n-$ $1), 2 /(n-1), \cdots, 1\}$, and to give:

$$
u_{i}=\arg \min _{u_{i} \in u_{d}}\left\|\mathbf{G}_{i}-\mathbf{C}\left(u_{i}\right)\right\|
$$

Thus, only segmented points $\mathbf{C}\left(u_{i}\right)$ on the continuous NURBS need to be communicated from the organising robots. Note that, depending on the number and length of NURBS for the case of multiple target entrapping patterns, the segmentation size of $u$ needs to be varied accordingly. For instance, if there are two NURBS curves for two separate entrapping patterns, the segmentation size is a half compared to the case for one curve so that robots can be equally distributed to each pattern.

\section{NumERICAL Simulations}

Numerical simulations have been performed using scenarios containing static/moving targets as well as a moving obstacle to validate the feasibility and benefits of the proposed algorithm. The number of robots used in the simulation is 20. Regulatory parameters for the upper layer of the EHGRN are those obtained from the previous section, and for the lower layer $a=6.4986, m=4.2417, c=9.9003$, $r=4.2886$, and $b=3.5392$ obtained by an evolutionary optimisation explained in [25]. In addition, the maximum speed of the robots is bounded by $0.8 \mathrm{~m} / \mathrm{s}$ considering the robots' physical capability. The distances to be maintained relative to the target and the obstacle set to $d_{\min }=2$, $d_{\max }=4$ and $d_{\min }^{\text {obs }}=4$. Slope values for the sigmoid in the fitness function in Eq. (7) are $k_{1}=1, k_{2}=2$, and $k_{3}=2$.

The entrapping of two stationary targets with an incoming obstacle is firstly tested as shown in Fig. 4. From their random initial locations (Fig. 4(a)), the robots are guided into the generated pattern by the upper layer of the EHGRN to entrap targets while keeping the constraints of being not too close or far away from them. As the obstacle gets closer, the entrapping pattern changes adaptively, and the robots are guided into the new position accordingly. Once the obstacle gets further away, robots return to the previous target entrapping pattern.

Fig. 5 shows the comparison between the previous and the proposed obstacle avoidance methods for the same scenario with an incoming moving obstacle. Fig. 5(a) is done by local reactive control by the lower layer only, similar to the previous work [25] which uses a diffusion term to avoid the collision as in Eq. (14). As an obstacle gets closer, some of the robots which lie within the effective range of the obstacle (illustrated by the red dash) are pushed away from their original desired positions, while the remaining robots (outside of the obstacle range) hold the position to entrap the targets. Thus, this scheme leads some of the robots to avoid the obstacle only, but not carrying out the original target entrapping task for a while. On the other hand, in Fig. 5(b), the effect of the obstacle is considered in the upper layer as well as the lower layer resulting in generating an adaptive pattern, desired positions for the whole robot swarm change continuously reflecting environmental changes.

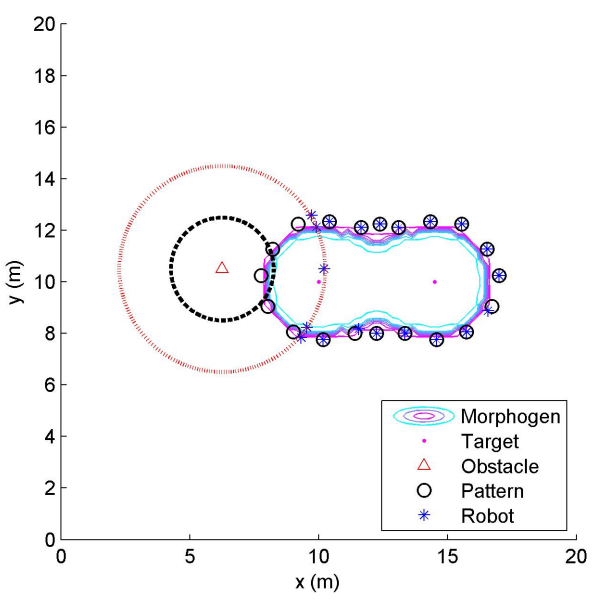

(a) Local reactive control by the lower layer

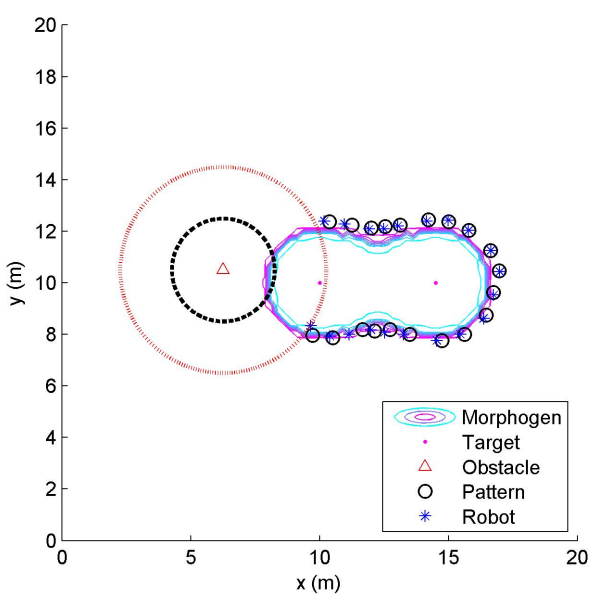

(b) Adaptive pattern generation by the upper layer

Fig. 5. Comparison between different obstacle avoidance methods.

Lastly, Fig. 6 shows the entrapping pattern separation as targets move away from each other. By the $k$-means clustering algorithm and cluster analysis as explained in the previous section, the number of entrapping patterns is determined, and the robots are distributed to the patterns accordingly. All these simulations verify the robustness and adaptability of the proposed algorithm to the environmental changes such as target and obstacle movement. 


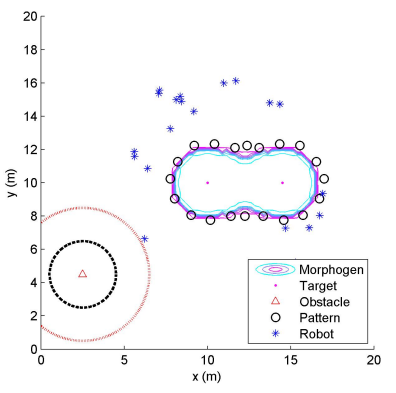

(a) $\mathrm{T}=0 \mathrm{~s}$

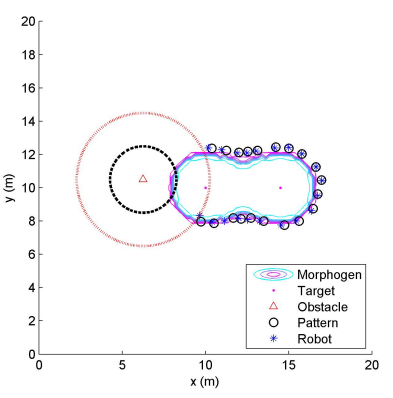

(d) $\mathrm{T}=18 \mathrm{~s}$

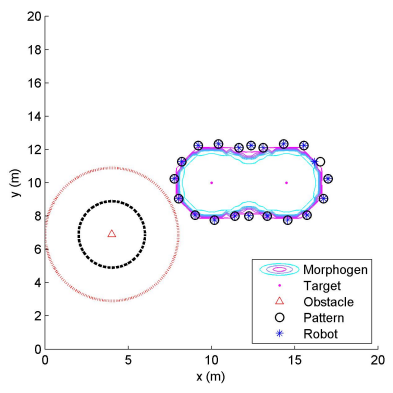

(b) $\mathrm{T}=6 \mathrm{~s}$

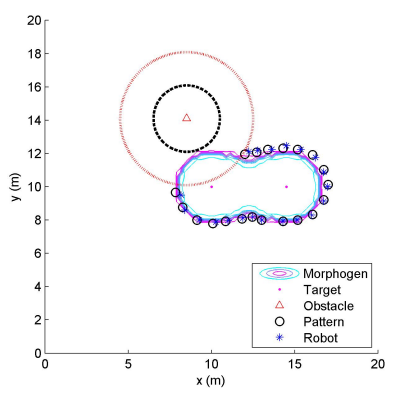

(e) $\mathrm{T}=27 \mathrm{~s}$

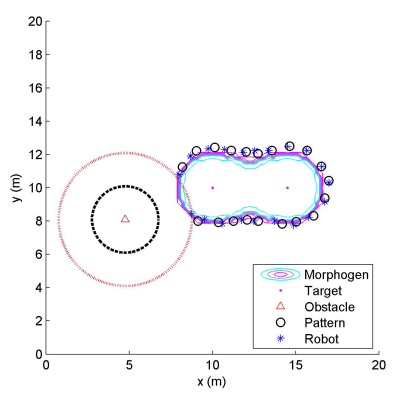

(c) $\mathrm{T}=12 \mathrm{~s}$

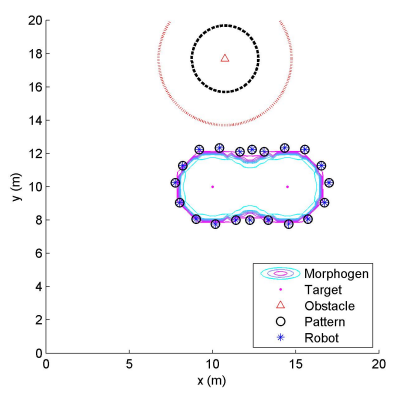

(f) $\mathrm{T}=36 \mathrm{~s}$

Fig. 4. Entrapping of two targets with a moving obstacle.

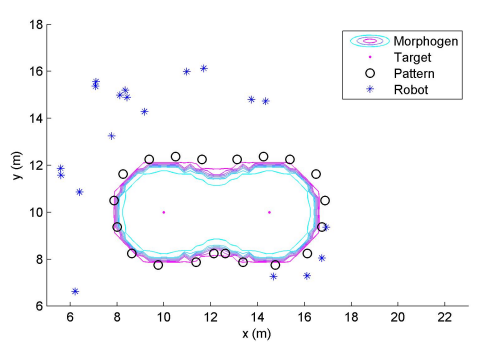

(a) $\mathrm{T}=0 \mathrm{~s}$

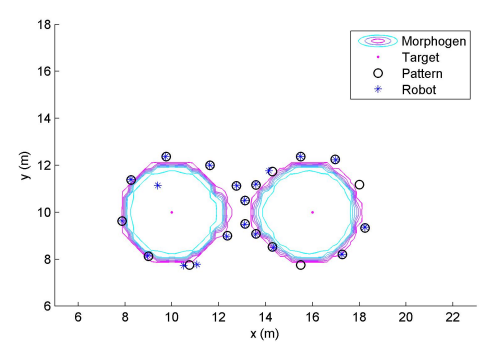

(d) $\mathrm{T}=18 \mathrm{~s}$

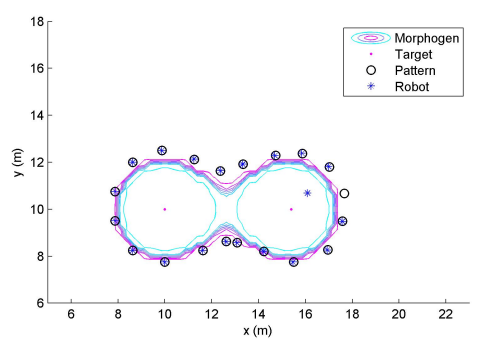

(b) $\mathrm{T}=6 \mathrm{~s}$

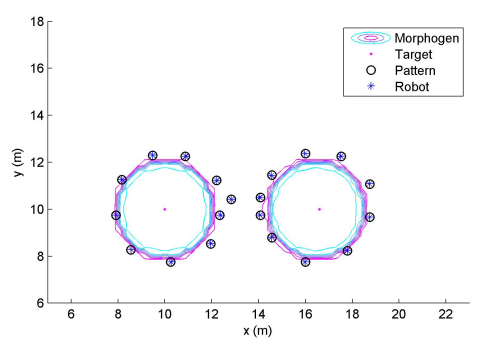

(e) $\mathrm{T}=27 \mathrm{~s}$

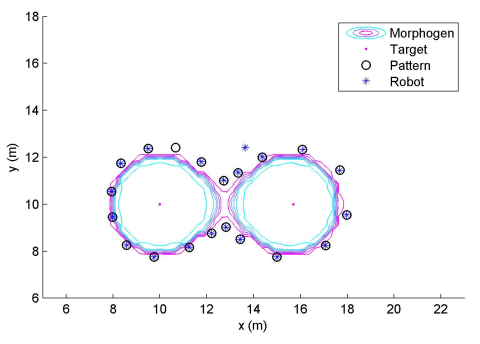

(c) $\mathrm{T}=12 \mathrm{~s}$

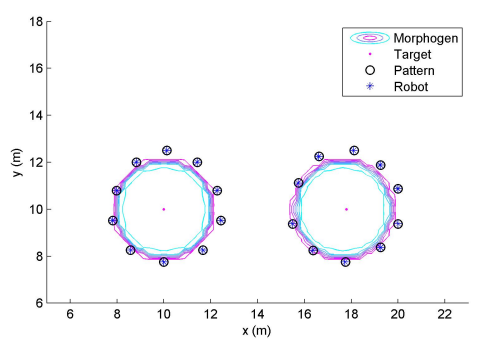

(f) $\mathrm{T}=36 \mathrm{~s}$

Fig. 6. Entrapping pattern separation as targets move away from each other. 


\section{CONCLUSIONS}

This paper has presented a morphogenetic approach to pattern formation for entrapping targets using swarm robots. The proposed evolving GRN framework improves the flexibility of the pattern generation to be applied for various tasks as it has an open structure of gene regulation to some extent. In addition, by introducing obstacles as one of environmental input sources along with that of targets, we address the weakness of the previous H-GRN pattern not being adaptable to obstacles in the sense that a target entrapping pattern itself changes as an obstacle approaches. Numerical simulations considering static/moving targets and obstacles have demonstrated that the proposed approach is able to automatically generate complex patterns highly adaptable and robust to dynamic and unknown environments. As future work, a proof-of-concept experiment will be performed to evaluate the proposed pattern formation algorithm using epuck education robots [39] in a real world. A fully open structure for evolving $\mathrm{H}-\mathrm{GRN}$ (i.e. the number of genes is not fixed) including the lower layer will be considered to be a more flexible and adaptable framework for various applications using swarm robots.

\section{REFERENCES}

[1] J. Pugh and A. Martinoli. Inspiring and modeling multi-robot search with particle swarm optimization. In IEEE Swarm Intelligence Symposium, 2007.

[2] E. Wong, F. Bourgault, and T. Furukawa. Multi-vehicle Bayesian search for multiple lost targets. In International Conference on Robotics and Automation, Barcelona, Spain, April 2005.

[3] M. Rubenstein, A. Cabrera, J. Werfel, G. Habibi, J. McLurkin, and R. Nagpal. Collective transport of complex objects by simple robots: Theory and experiments. In 12th International Conference on Autonomous Agents and Multiagent Systems, Minnesota, USA, May 2013.

[4] I. Maza, F. Caballero, J. Capitan, J.R. Martinez de Dios, and A. Ollero. A distributed architecture for a robotic platform with aerial sensor transportation and self-deployment capabilities. Journal of Field Robotics, 28(3):303-328, 2011.

[5] H. Oh, S. Kim, J. Suk, and A. Tsourdos. Coordinated trajectory planning for efficient communication relay using multiple UAVs. In 19th IFAC Symposium on Automatic Control in Aerospace, Wurzburg, Germany, Sep. 2013.

[6] H. Oh, S. Kim, H.-S. Shin, A. Tsourdos, and B. White. Coordinated standoff tracking of groups of moving targets using multiple UAVs. In 21st Mediterranean Conference on Control and Automation, Crete, Greece, June 2013.

[7] R.W. Beard, J. Lawton, and F.Y. Hadaegh. A coordination architecture for spacecraft formation control. IEEE Transactions on Control Systems Technology, 9(6):777-790, 2001.

[8] J.R. Lawton, R.W. Beard, and B.J. Young. A decentralized approach to formation maneuvers. IEEE Transactions on Robotics and Automation, 19(6):933-941, 2003.

[9] T. Balch and R.C. Arkin. Behavior-based formation control for multirobot teams. IEEE Transactions on Robotics and Automation, 14(6):926-939, 1998.

[10] L. Consolini, F. Morbidi, F. Prattichizzo, and M. Tosques. Leaderfollower formation control of nonholonomic mobile robots with input constraints. Automatica, 44(5):1343-1349, 2008.

[11] H. Oh, S. Kim, A. Tsourdos, and B. White. Decentralised standoff tracking of moving targets using adaptive sliding mode control for UAVs. Journal of Intelligent and Robotic Systems, 2013. DOI:10.1007/s10846-013-9864-0, in press.

[12] M. Hsieh, V. Kumar, and L. Chaimowicz. Decentralized controllers for shape generation with robotic swarms. Robotica, 26(5):691-701, 2008 .
[13] A.R. Pereira and L. Hsu. Adaptive formation control using artificial potentials for eulerlagrange agents. In 17th IFAC world congress, 2008.

[14] W.-M. Shen, P. Will, and A. Galstyan. Hormone-inspired selforganization and distributed control of robotic swarms. Autonomous Robots, 17:93-105, 2004.

[15] H. Xu, H. Guan, A. Liang, and X. Yan. A multi-robot pattern formation algorithm based on distributed swarm intelligence. In International Conference on Computer Engineering and Applications, 2010.

[16] H. Guo, Y. Meng, and Y. Jin. A cellular mechanism for multi-robot construction via evolutionary multi-objective optimisation of a gene regulatory network. BioSystems, 98:193-203, 2009.

[17] Y. Jin and Y. Meng. Morphogenetic robotics: An emerging new field in developmental robotics. IEEE Transactions on Systems, Man, and Cybernetics, Part C: Applications and Reviews, 41(2):145-160, 2011.

[18] C. Belta and V. Kumar. Abstraction and control for groups of robots. IEEE Transactions on Robotics, 20(5):865-875, 2004.

[19] C.C. Cheah, S.P. Hou, and J.J.E. Slotine. Region-based shape control for a swarm of robots. Automatica, 45:2406-2411, 2009.

[20] S.P. Hou and C.C. Cheah. Multiplicative potential energy function for swarm control. In IEEE/RSJ International Conference on Intelligent Robots and Systems, pages 4363-4368, St. Louis, USA, Oct. 2009.

[21] H. Sayama. Robust morphogenesis of robotic swarms. IEEE Computational Intelligence Magazine, 5(3):43-49, 2010.

[22] R. Doursat, H. Sayama, and O. Michel. A review of morphogenetic engineering. Natural Computing, 12:517-535, 2013.

[23] S. Kondon and T. Miura. Reaction-diffusion model as a framework for understanding biological pattern formation. Science, 329:1616-1620, 2010.

[24] S. Basu, Y. Gerchman, C.H. Collins, F.H. Arnold, and R. Welss. A synthetic multicellular system for programmed pattern formation. Nature, 434:1130-1134s, 2005.

[25] Y. Jin, H. Guo, and Y. Meng. A hierarchical gene regulatory network for adaptive multirobot pattern formation. IEEE Trans. on Systems, Man, And Cybernetics-Part B: Cybernetics, 42(3):805-816, 2012.

[26] D. Erwin and E. Davidson. The evolution of hierarchical gene regulatory networks. Nature Reviews Genetics, 10(2):141-148, 2009.

[27] Y. Meng and H. Guo. A gene regulatory network based framework for self-organization in mobile sensor networks. In IEEE World Congress on Computational Intelligence, Brisbane, Australia, June 2012.

[28] C.M. Bishop. Neural Networks for Pattern Recognition. Oxford University Press, 1995.

[29] M. MAMEI, M. VASIRANI, and F. ZAMBONELLI. Experiments of morphogenesis in swarms of simple mobile robots. Applied Artificial Intelligence, 18:903-919, 2004.

[30] H. De Jong. Modeling and simulation of genetic regulatory systems: A literature review. Journal of Computational Biology, 9(1):67-103, 2002.

[31] T. Bouwmeester. The spemann-mangold organizer: The control of the fate specification and morphogenetic rearrangements during gastrulation in xenopus. International Journal of Developmental Biology, 45:251-258, 2001.

[32] R. Milo, S. Shen-Orr, S. Itzkovitz, N. Kashtan, D. Chklovskii, and U. Alon. Network motifs: Simple building block of complex networks. Science, 298:824-827, 2002.

[33] N. Kashtan and U. Alon. Spontaneous evolution of modularity and network motifs. Proceedings of National Academy of Sciences, 102:13773-13778, 2005.

[34] L. Piegl and W. Tiller. The NURBS Book. New York: Springer-Verlag, 1997.

[35] N. Hansen and A. Ostermeier. Completely derandomized selfadaptation in evolution strategies. Evolutionary Computation, 9(2):159-195, 2001.

[36] H. Guo, Y. Jin, and Y. Meng. A morphogenetic framework for selforganized multirobot pattern formation and boundary coverage. ACM Trans. on Autonomous and Adaptive Systems, 7(1):15:1-23, 2012.

[37] Y. Meng, H. Guo, and Y. Jin. A morphogenetic approach to flexible and robust shape formation for swarm robotic systems. Robotics and Autonomous Systems, 61:25-38, 2013.

[38] I. Salazar-Ciudad, J. Jernvall, and S. Newman. Mechanisms of pattern formation in development and evolution. Development, 130(10):20272037, 2003.

[39] http://www.e-puck.org/. 